\title{
New fast-relaxed liquid crystal materials for optical communication networks
}

\author{
Yu. Garbovskiy, A. Sadovenko, A. Koval'chuk, G. Klimusheva, S. Bugaychuk \\ Institute of Physics, NAS of Ukraine, 46, prospect Nauky, 03028 Kyiv, Ukraine \\ Phone: 525-78-41; e-mail: klimush@iop.kiev.ua
}

\begin{abstract}
Recording the dynamic holograms with microsecond relaxation times under action of laser pulses was obtained in composites based on the novel class of liquid crystals (LC), namely in ionic metal-alkanoates. Holographic parameters of the recording and relaxation characteristics were studied for doped lyotropic ionic LC and sandwichlike cells with photo-sensitive impurities for purposes of real-time dynamic holography applications. The thin cells demonstrate high-velocity dynamic grating recording under laser pulses both of nanosecond and picosecond durations at the visible wavelengths. The cells exhibit a fast temperature relaxation time (with the time constant $30 \mu$ s for the store heat density more than $50 \mathrm{~kJ} / \mathrm{s}$ ). Ionic lyotropic smectic LCs possess a high intrinsic anisotropic conductivity as compared with other LCs - dielectrics. To explain the relaxation mechanisms in ionic smectic LC matrix, the temperature dependences of the electro-conductivity have been investigated. The charge currier mobility and activation energy in cells were estimated. The mechanism of high-velocity resonance nonlinearity due to the saturation of excited states in photosensitive centers and mechanisms of the grating erasure connected with charge transport in the ionic LC matrix were discussed.
\end{abstract}

Keywords: ionic smectic liquid crystals, dynamic holographic recording, liquid crystal electrolyte, high-velocity process.

Manuscript received 23.11.05; accepted for publication 15.12.05.

\section{Introduction}

Nowadays, known is a lot of different nonlinear optical materials used for the holographic recording [1]. The media with resonance nonlinearity has much interest for fast and high-efficient recording and processing the information. The liquid [2], the liquid crystal (LC) [3] and the rigid [4] dye solutions belong to them. However, strong thermal and long-time orientation effects are often observed in these matrixes, and they lead to restrictions in the spatial resolution and to increased relaxation times of the holographic recording.

The recording of dynamic holographic gratings with fast relaxation (erasure) under nanocesond laser pulses is obtained in the novel class of ionic LCs, namely, in the ionic mesogenes of metal alkanoate with photosensitive impurities. Researches of nonlinear-optical properties of the sandwich-like cells and particular features of the electric conductivity in pure and impurity ionic lyotropic liquid crystals (ILLC) are presented in the paper.

To explain the role of the ILLC matrix in the grating relaxation process, we investigated the electric conductivity of the ILLC in the temperature range of 292 to $333 \mathrm{~K}$. We obtained the high value of the conductivity for comparison with other LC dielectrics. We determined the activation energy of the ILLC. An estimation of the diffusion characteristics for charge carriers was made. The mechanisms of the holographic recording in sandwich-like cells as well as the mechanism of the conductivity in new materials (pure and impurity ILLC) were discussed.

\section{Materials}

ILLC are formed by water solution of metal alkanoates and they have the spatial structure similar to smectic A. The molecules in the ILLC have the bi-layer packing of the hydrophobic alkanoate chains consisting of the metal cations, carboxyl anions and water molecules. Polymethine dyes are easily solved in ILLC.

In this work, we investigate the sandwich cells consisting of a thin film of a dye (a polymethine dye relating to either an anion or cation types) and a layer of the planar oriented ILLC. The ILLC is the water solution of the potassium caproate in the proportion 1:1. The sandwich cells are prepared in the following way. We take two glass plates. A thin layer of a dye solution is placed onto one plate. After evaporation of the solvent, 
the dye polycrystalline plate is covered by an ILLC layer, covered with the second plate, and all edges are glued to avoid evaporation of water. The cells have the thickness of 20 to $30 \mu \mathrm{m}$.

To prepare the samples for investigation of electric properties, an ILLC layer was placed between glass plates. As spacers, we used two metal strips (made of $\mathrm{Cu}$ and $\mathrm{Ni}$ ) that served as electrodes and preset the thickness of the ILLC layer. The edges of this cell were glued. The thickness of these cells varied from $25 \mu \mathrm{m}$ up to $1.5 \mathrm{~mm}$.

\section{Holographic properties of ILLC}

The method of two-beam mixing by pulse Nd:YAP laser operated at the double frequency is used to investigate the holographic grating recording. The parameters of the laser were as follows: the wavelength $\lambda=539.8 \mathrm{~nm}$, the pulse duration $\tau=20 \mathrm{~ns}$, the pulse repletion frequency $v=3 \mathrm{~Hz}$. The output laser radiation was linearly polarized in the horizontal plane. The laser beam went through a diaphragm of the diameter $1 \mathrm{~mm}$ and a splitting system, so that two laser beams were converged on an investigated cell. The intensities of both the beam incident on the cell and the diffracted orders were detected by photodiodes in the self-diffraction regime. To investigate the time relaxation characteristics of the dynamic gratings, we use a probe non-polarized beam from a continuous $\mathrm{He}-\mathrm{Ne}$ laser.

In sandwich cells, the self-diffraction is revealed already after one laser pulse. Several diffraction orders are observed during this process. This fact proves that a thin grating is formed (the Raman-Nath regime). We have found that solid dye films provide the recording of permanent gratings, and only covering them with the ILLC layer allows one to obtain the dynamic grating recording with fast erasure time.

To determine the dependence of the absorption in the cells on the radiation energy density $E$ at $\lambda=539.8 \mathrm{~nm}$, we investigated the changes of the optical density $D$ in the cells versus $E$ during increase of $E$ and, in the reverse direction, during decrease of $E$ (Fig. 1). We found the brightening effect in the sandwich cells: the optical density drops on the average from the value 2 to 1 in the range of 0.1 to $1 \mathrm{~mJ} / \mathrm{mm}^{2}$. But after action of the laser irradiation, the optical density of the cells does not return to its initial value. This fact shows that in addition to evident reversible changes in the absorption, there are also some irreversible ones connected with photochemical transformation of dye molecules under powerful laser irradiation. In this process, the sandwich cells with the anion dye turn out to be stable to the laser irradiation in comparison with the cells containing the cation one.

In Fig. 2, shown is the dependence of the energy density in the first diffracted order of the self-diffraction $\left(E_{+1}\right)$ versus the input energy density $(E)$ in the sandwich cell with the anion dye. The grating period was fixed and equaled to $16 \mu \mathrm{m}$. One can see that the increasing $E_{+1}$

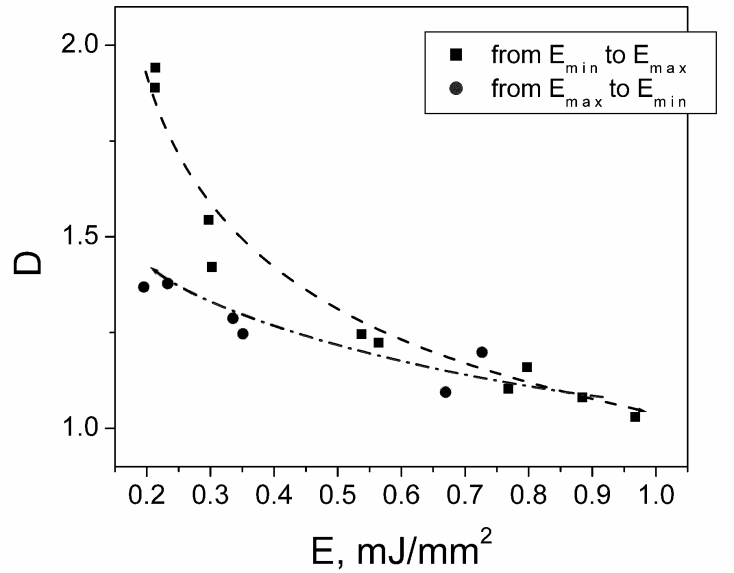

Fig. 1. The changes of the optical density versus the energy density in one laser pulse in a sandwich-like cell ILSLC with the anion dye.

with growing $E$ obey the cubic law. The similar dependence is observed for the cell with the cation dye (Fig. 3). The experimental results testify that our cells have the cubic nonlinearity that is typical for resonance media. We investigated the diffraction efficiency depending on the grating period. The efficiency of the self-diffraction demonstrates a slight increase in the range from 3 to $30 \mu \mathrm{m}$ versus the grating period. In contrast to impurity nematics [5], the sandwich cells provide a more effective recording for small periods, which leads to increasing the spatial resolution of the holographic recording in the medium.

The kinetics of dynamic grating relaxation starting after $5 \mu \mathrm{s}$ of nanosecond recording laser pulses is investigated for the energy density $E=1 \mathrm{~mJ} / \mathrm{mm}^{2}$ and grating period $\Lambda=16 \mu \mathrm{m}$. The experiment allows us to define the real time of the complete erasure of gratings. The typical behavior of the observed relaxation curve is shown in Fig. 4. One can see that vanishing the secondary thermal grating obeys the exponential law with the characteristic time of the order of $30 \mu \mathrm{s}$.

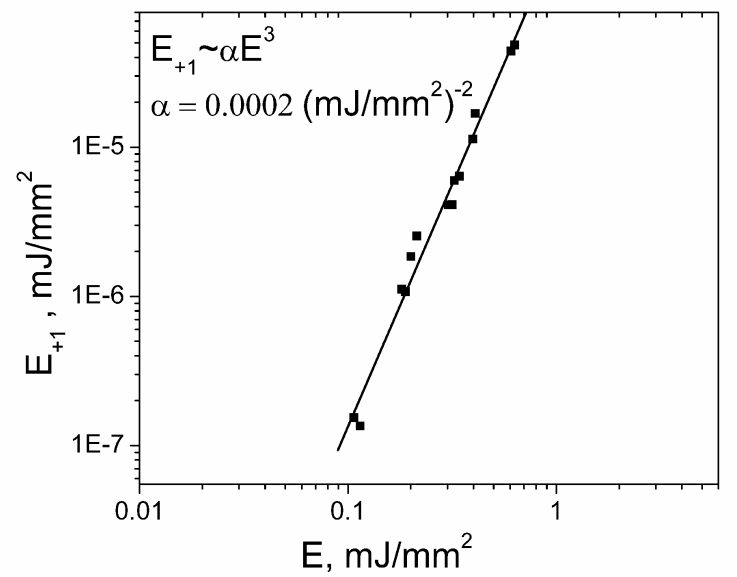

Fig. 2. The measured energy density of the first diffracted order of the self-diffraction versus the energy density in one laser pulse in a sandwich-like cell with the anion dye. 


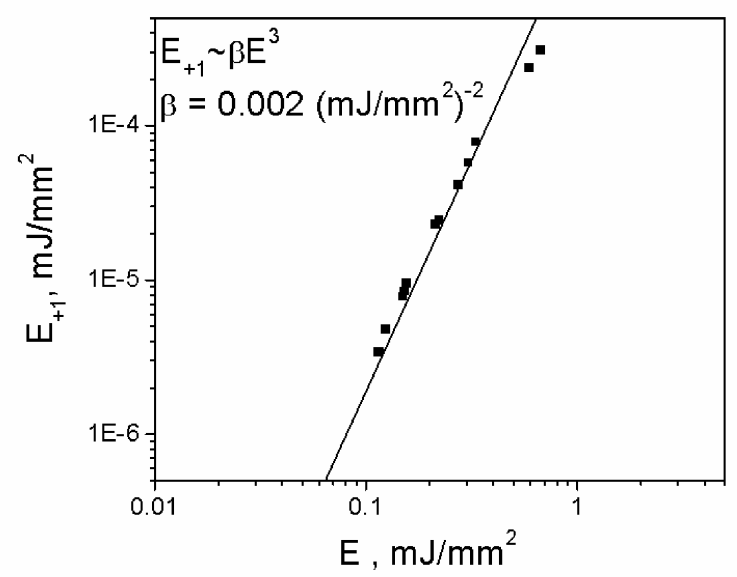

Fig. 3. The measured energy density of the first diffracted order of the self-diffraction versus the energy density in one laser pulse in sandwich-like cell with the cation dye.

The obtained main holographic characteristics in sandwich cells (consisted of a thin dye film and an ILLC layer) prove that the primary mechanism of the grating recording is the resonance nonlinearity caused by the effect of the saturation absorption in the dye molecules. The ILLC matrix provides a fast erasure of the secondary heat grating, which is created after nonradiative relaxation of excited impurity molecules. Local heating in the cells is a result of dye molecule relaxation from the excited state to the ground one. This process lasts within nanoseconds. In the case when there is a solid dye film alone in the cell, the fast temperature increase leads to melting and evaporation of photosensitive molecules what can be seen from the interference peaks of the light pattern. A permanent relief grating is formed as the result of this process. Just the ILLC layer covered the dye film in sandwich cells provides fast heat dissipation and makes the recording to be the dynamical one.

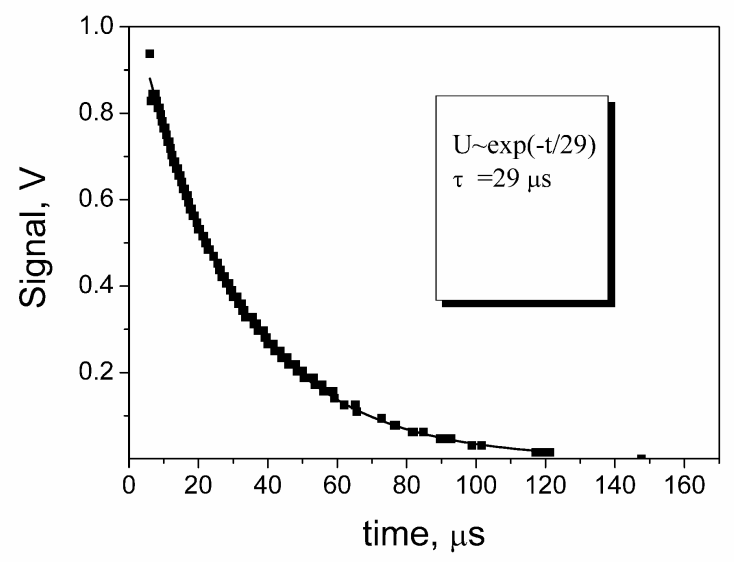

Fig. 4. The kinetics of the probe beam intensity in the first diffracted order for a sandwich-like cell with the cation dye.

\section{Electrical properties of ILLC}

To find the explanation of grating recording relaxation, we study the dependence of conductive properties of ILLC versus the temperature. The electric conductivity was measured using the oscilloscopic technique [6, 7]. A voltage signal had a triangular shape with the pick amplitude 0.10 to $0.25 \mathrm{~V}$. The signal frequency was changed within the range of 0.1 to $10^{6} \mathrm{~Hz}$. The electroconductivity was measured in the range of $10^{4}$ to $10^{6} \mathrm{~Hz}$, where the resistance of the samples does not depend on the frequency. To make the temperature measurements, the samples were placed into a thermostat that was especially designed for these experiments. The temperature values were determined using a thermocouple with the accuracy $0.5 \mathrm{~K}$.

The phase transition of ILLC is absent in the investigated temperature range $(292-333 \mathrm{~K})$. The ILLC in our cells have a planar domain structure. The direction of electric field is mainly along alkyl chains to be perpendicular to the electrostatic layers of ILLC. In the experiments, we measured a geometric parameter of the cell $k=\ell /(d \cdot z)$ by varying its thickness $d$, the length of metal electrode-spacers $z$ and the distance between them $\ell$. The conductivity $\sigma_{\text {a.c. }}$ was calculated from the measured value of the volume resistance $R$ and the known geometric parameter $k$ of the cell by using the formula: $\sigma_{\text {a.c. }}=k / R$. We obtained the value of the conductivity at the room temperature $\sigma_{\text {a.c. }}=$ $10 \ldots 11 \mathrm{Ohm}^{-1} \cdot \mathrm{m}^{-1}$. A typical temperature dependence of the volume resistance for the sample $R$ is shown in Fig. 5. One can see that the experimental points are very well approximated by Arrhenius' equation [8]. From the slope of the line in Fig. 5, we determine the conductivity activation energy $E_{a}\left(E_{a}=0.21 \mathrm{eV}\right)$.

Earlier it was established [9] that the anisotropy of the conductivity is the distinguishing feature for thermotropic ionic LCs of metal alkanoates. One has to expect a similar anisotropic conductivity for ILLC that has the same smectic structure as the thermotropic mesophases. To prove this statement, the conductivity in thick samples $(1.5 \mathrm{~mm})$ with random domain structure in the volume has been investigated. We found that any changes of the electric field direction do not change the conductivity of the volume samples, i.e., the "thick" sample possesses isotropic electric properties. The conductivity of such samples is 32 to $36 \mathrm{Ohm}^{-1} \cdot \mathrm{m}^{-1}$ at the room temperature what is higher as compared with the planar orientated thin cells. We determined the conductivity activation energy $E_{a}=0.16 \ldots 0.17 \mathrm{eV}$ from the temperature dependences of the volume resistance in non-oriented samples. Nevertheless, the fact of the significant difference in the conductivity (by 3 to 3.5 orders) between non-oriented samples $\left(32 \ldots 36 \mathrm{Ohm}^{-1} \cdot \mathrm{m}^{-1}\right)$ and planar oriented cells $\left(10 \ldots 11 \mathrm{Ohm}^{-1} \cdot \mathrm{m}^{-1}\right)$ (where the electric field is applied on the average not along the electrostatic layer but 


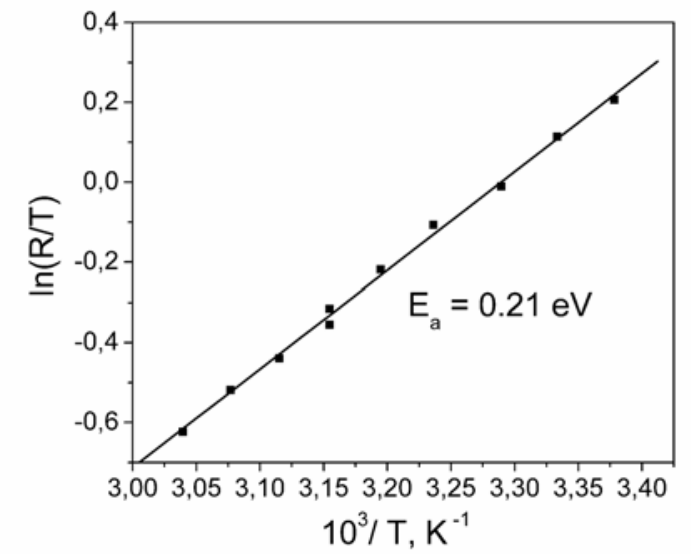

Fig. 5. The volume resistance of pure ILSLC versus an inverse temperature.

perpendicular to them) is indicative of the existence of the anisotropic conductivity in ILLC.

Similar experimental data for the conductivity and the activation energy have been obtained for impurity ILLC with anion dye. Since the high own intrinsic conductivity of ILLC exists, doping by the anion dye does not lead to essential changes of those electrical properties.

We would like to note that one has to use only metallic electrodes to measure conductivity in ILLC. Applying the ITO electrodes that are usually used to study electric properties in dielectrics gives values several orders smaller for well conductive media.

We investigated also the charge carrier mobility in ILLC. For this purpose, we use the method of the inverse polarity [10]. The sample was connected with a source of the constant voltage (2 B) that polarize the sample. When the current passing through the sample reaches a constant value, this source is disconnected and other source gives inverse pulling voltage $(1.5 \mathrm{~B})$ to the sample. The signal from the sample input to a grapher that fixed the dependence with a maximum for the current value. Being based on the position of the maximum and the relation $\mu=d^{2} /(U \cdot t)$, where $d$ is the sample thickness, $U$ is the voltage, $t$ is the time of the maximum current, we found the value of the charge carrier mobility $\mu$ in ILLC. We determined the charge carrier mobility $\mu \approx 2 \cdot 10^{-7} \mathrm{~m}^{2} / \mathrm{V} \cdot \mathrm{s}$.

Our experimental researches and estimations permits to make some conclusions about a possible mechanism of the conductivity in ILLC as well as its role in the process of the dynamic grating relaxation. The high value $\sigma_{\text {a.c. }}\left(10\right.$ to $\left.36 \mathrm{Ohm}^{-1} \cdot \mathrm{m}^{-1}\right)$ and absence of the effect of the electrolytic treatment of ILLC prove the existence of mainly intrinsic ionic conductivity. Taking the ILLC structure into consideration, one can assume that the main contribution to the conductivity is provided by potassium cations that can easy move along electrostatic layers of ILLC in contrast to carboxyl- anions because they have a size that is tens times greater than the potassium cation one.

The obtained results about the small activation energy of the conductivity in ILLC are important to understand a role of the ILLC matrix in the process of the dynamic grating erasure. As it was noted above, the local heating occurs during dynamic grating recording on the interference peaks as the result of relaxation of excited dye molecules. During this process, a thermal activation of potassium cations in ILLC takes place. The activated charge can move over the distance of fractions of a micrometer over the time of several microseconds, to provide in such a manner a partial heat removal from the places of local heating.

\section{Conclusions}

For the first time, the holographic recording of dynamic grating was obtained in materials based on conductive ILLC that provide fast relaxation of recorded holograms in the microsecond range. Our experimental results prove that the primary mechanism of grating recording in sandwich cells (consisted of a thin dye film covered with the ILLC layer) is caused by a resonance nonlinearity of the saturation absorption in two-level electronic transitions in dye molecules. Under intensive laser irradiation with the frequency close to the frequency of the electronic transition, the occupance of the excited level takes place. Also the brightening effect is observed as the result of this process.

From our experimental researches of electric properties, the activation character of the temperature dependences of the conductivity in ILLC is ascertained. We found that the ILLC belongs to systems that are sensitive to low electric fields and possesses anisotropic high-valued intrinsic ionic conductivity (from 10 to $\left.36 \mathrm{Ohm}^{-1} \cdot \mathrm{m}^{-1}\right)$. The conductivity of the investigated ILLC is 5-10 orders higher as compared with the traditional molecular LCs. Obtained values of the conductivity are close to those in liquid or solid electrolytes having the similar compounds, but in contrast to them ILLC are a LC electrolyte.

Investigated specific properties of ILLC show that these materials belong to media that provide fast erasure of thermal gratings being formed after relaxation of excited molecules of dyes. This fact follows from the possibility of thermal activation of potassium cations due to existence of a close contact between an ILLC layer and a dye film. Optimization of the compound of ILLC based cells permits to obtain new perspective materials possessing high-velocity effective dynamical recording with fast erasure time.

\section{References}

1. M.I. Eichler, P.G. Gunter, D.W. Pohl, Laser-induced dynamic grating. Springer-Verlag, Berlin Heidelberg (1986). 
2. Ye.A. Tikhonov, M.T. Shpak, Nonlinear optical phenomena in organic compositions. Naukova Dumka, Kiev (1979) (in Russian).

3. H.J. Eichler, D. Grebe, R. Macdonald, A.G. Iljin, G.V. Klimusheva, L.I. Zagainova // Optical Materials, 2, p. 201-208 (1993).

4. N. Tomova, V. Dragostinova, L. Nikolova, I. Radoslavova, T. Todorov // J. Signal AM, 9(5), p. 373-379 (1981).

5. C.V. Serak, A.A. Kovalev, A.V. Agashkov // Zhurn. Tekhnich. Fiziki 70, No 7, p. 44-51 (2000) (in Russian).
6. F.J. Twarowski, A.C. Albrecht // J. Chem. Phys., 70, N 5, p. 2255-2261 (1979).

7. A. V. Koval'chuk // Ukr. Fiz. Zhurn. 41, p. 991-998 (1996) (in Ukrainian).

8. A. Lid'jard, Ionic conductivity of crystals. Inostrannaya Literatura, Moscow (1962) (in Russian).

9. J.J. Duruz, A.R. Ubbelohde // Proc. R. Soc. London A 347, p. 301-310 (1976).

10. N.I. Gritsenko, N.V. Moshel' // Ukr. Fiz. Zhurn. 25, p. 1830-1835 (1980) (in Russian). 